\title{
Licence Profile: Apache License, Version 2.0
}

\author{
Andrew Sinclair ${ }^{a}$ \\ (a) Legal Counsel, Canonical USA, Inc.
}

DOI: $\underline{10.5033 / \text { ifosslr.v2i2.42 }}$

\begin{abstract}
The Apache License, Version 2.0 is a widely used permissive open source software license which builds on the terms of other permissive open source licenses to produce a license with similar principles to those of licenses like the BSD License and MIT License, but with expanded terms such as a more developed copyright licence grant and a patent licence grant. This article is short overview of the license.
\end{abstract}

\author{
Keywords \\ Apache License; Law; information technology; Free and Open Source \\ Software \\ Info \\ This item is part of the Articles section of IFOSS L. Rev. For more \\ information, please consult the relevant section policies statement. \\ This article has been independently peer-reviewed.
}

As a permissive open source licence (an open source licence that features broad permissions and no "copyleft" provision), the Apache License, Version 2.0 (hereinafter referred to as the "Apache License") has similar legal effects to those of licences like the BSD License, MIT License and historic permission notices. The Apache License is not as popular as these if measured by number of projects, but it is a significant open source licence due to the importance of the projects that use it, such as the Apache HTTP server, which is used by over $50 \%$ of all web servers. ${ }^{2}$ The Apache License is also more developed than simpler licences like the BSD License. As a result, it relies less on common community interpretation to overcome potential legal ambiguities, while still retaining the core terms of a permissive open source licence. ${ }^{3}$

1 http://www.apache.org/licenses/LICENSE-2.0.html [hereinafter Apache License]

2 http://news.netcraft.com/archives/2010/11/05/november-2010-web-server-survey.html

3 For a discussion of potential legal ambiguities of the BSD License, see Sinclair, Andrew (2010) 'Licence Profile: BSD', IFOSS L. Rev., 2(1), pp 1 -6, DOI: $10.5033 /$ ifosslr.v2i1.28 


\section{Licence history and use}

The current version of the Apache License is the third iteration of licence. The history of the licence aids our understanding the current version: The original version was very similar to the original BSD License ${ }^{4}$, and the Apache Software Foundation updated the original to remove the required advertising clause following the same update of the BSD License. ${ }^{5}$ In 2004, the Apache Software Foundation approved version 2.0, which retains the same principles as the earlier versions, but expands and clarifies their terms. ${ }^{6}$

While it can be useful to gauge the importance of a licence by its popularity in terms of number of open source projects, such a gauge only measures one aspect of a licence's significance. In the case of the Apache License, popularity numbers have the potential to mislead. The Apache License comprises approximately $2 \%$ of the projects hosted on SourceForge ${ }^{7}$, but as the licence for all Apache Software Foundation projects ${ }^{8}$ and Android $^{9}$, it is a licence that governs significant open source projects.

\section{Parsing the licence}

The Apache License consists of a series of clauses covering the licence terms followed by a short appendix setting out a standard format of a copyright and licence notice. This profile will discuss a few of the key clauses.

\section{Copyright licence grant}

As would be expected, the Apache License has a broad and very permissive copyright licence grant. It is surely written with the US Copyright Act in mind, as the grant language runs parallel to statutory rights enumerated in Section 106 of the US Copyright Act. ${ }^{10}$ The grant includes the rights to, "reproduce, prepare Derivative Works of, publicly display, publicly perform, sublicense, and distribute the Work and such Derivative Works in Source or Object form." ${ }^{11}$ Listing each of the US Copyright Act's exclusive rights in copyrighted works provides a far less ambiguous grant, at least under US law, than that of the BSD License or versions 1.0 and 1.1 of the Apache License. Those licences all have the same very simple grant, "Redistribution and use in source and binary forms, with or without modification, are permitted ..."12

The copyright licence grants rights from each "Contributor". This is a defined term roughly meaning anyone with a copyright ownership interest in the work or part of it. This structure,

4 Compare the original BSD License (http://www.xfree86.org/3.3.6/COPYRIGHT2.html\#6) to the Apache License, Version 1.0 (http://www.apache.org/licenses/LICENSE-1.0)

$5 \mathrm{http}: / / \mathrm{www}$.apache.org/licenses/ (Stating, "The 1.1 version of the Apache License was approved by the ASF in 2000. The primary change from the 1.0 license is in the 'advertising clause' (section 3 of the 1.0 license); derived products are no longer required to include attribution in their advertising materials, only in their documentation.")

$6 \quad I d$.

7 http://www.oss-watch.ac.uk/resources/apache2.xml

8 http://www.apache.org/licenses/\#distributions

9 http://www.openhandsetalliance.com/android faq.html

10 United States Copyright Act, 17 U.S.C. \$ 106 (2009)

11 Apache License, supra note 1

12 http://www.opensource.org/licenses/bsd-license.php 
whereby the rights under the licence are granted from each copyright holder to each downstream licensee, is common in open source licensing, but it is not always explicit. One interesting feature of the Apache License grant is that it includes a right to sublicense. This may be intended to ensure that the Apache License is compatible with different licensing models. For example, if a licensee wanted to combine code licensed under the Apache License with code under a "copyleft" licence (a licence requires the resulting combination to be licensed under only that licence), that licensee may have no other choice but to sublicense the Apache License code under the "copyleft" licence. The sublicense right could also be useful in a proprietary licence context if a proprietary software vendor desires to sublicense Apache License code rather than simply passing the Apache License through to the end user or re-distributor. In many cases, however, there would not be a need to sublicense because the licence is granted directly from each "Contributor" to each licensee.

\section{Patent licence grant}

The Apache License expressly grants both a copyright licence and a patent licence to licensees. This is somewhat unusual among permissive open source licences, which do not usually mention patents. The delineation of the copyright and patent grants give clarity to the scope of licence which is absent in more simple examples like the BSD License and MIT License. Those licences are sometimes thought not to grant a patent licence, but their grant language does not mention copyright or patent. The licensee must look for clues in the context of the grant, such as references to the copyright statement or "copyright holders" elsewhere in the licence as well as community interpretation to assess whether a patent right is granted. The Apache License suffers no such ambiguity.

The patent licence grant of the Apache License, like the copyright licence grant, seems to be based on US statutory law. The licence includes the rights to make, use, sell, and import, which are the terms used in the US Patent Act. ${ }^{13}$ The licence, "applies only to those patent claims licensable by such Contributor that are necessarily infringed by their Contribution(s) alone or by combination of their Contribution(s) with the Work to which such Contribution(s) was submitted." ${ }^{14}$ One of the problems with patent licences in open source software is that the software is likely to change downstream. A narrow patent license may not cover certain future modifications or combinations of the software, but a broad patent licence may license more than the licensor intended. The Apache License takes a relatively narrow approach; its grant is limited to the contribution and the resulting combinations of that contribution with the rest of the work at the date of submission. This grant seems to exclude patents that would be infringed by modifications of the contribution, at least to the extent such patents would not be infringed by the original contribution. ${ }^{15}$ The licensee receives the grant from all contributors, but if a midstream contributor altered upstream code or combined it with the work in a new way, the downstream licensee may not have received all the applicable patent rights from each contributor.

\footnotetext{
13 United States Patent Act, 35 U.S.C. $\$ 271$ (2010)

14 Apache License, supra note 1

15 The Apache Foundation License FAQ supports this reading, "Q1: If I own a patent and contribute to a Work, and, at the time my contribution is included in that Work, none of my patent's claims are subject to Apache's Grant of Patent License, is there a way any of those claims would later become subject to the Grant of Patent License solely due to subsequent contributions by other parties who are not licensees of that patent. A1: No." (http://www.apache.org/foundation/licence-FAQ.html)
} 
The last sentence of the patent licence section is not actually part of the licence grant; it is a patent termination clause. If a licensee under the Apache License brings a patent claim alleging that a work under the Apache License infringes that licensee's patent, the Apache License ceases with respect to that licensee. This is a simple and relatively strong clause, as it applies to any patent litigation claim with respect to code under the Apache License and does not offer any resolution period (the termination is effective immediately upon the filing of the patent litigation claim). Similar patent termination clauses can be found in other open source licences; one example is the Mozilla Public License version 1.1 (hereinafter "MPL") ${ }^{16}$. The MPL's patent termination clause terminates a potentially more narrow set of rights: those granted by the party against whom the claim is asserted, and it provides, in some cases, a sixty day resolution period giving the party bringing the claim an opportunity to resolve the dispute before the licence terminates. ${ }^{17}$ This clause should discourage patent claims where a would-be plaintiff is benefiting from the use of the Apache Licensed software.

\section{Redistribution rights and conditions}

The Apache License sets out a number of terms specific to redistribution. ${ }^{18}$ In addition to terms requiring preservation of legal and attribution notices, a distributor must provide a copy of the Apache License to recipients and add prominent notices with respect to modified files. These terms would be expected of a licence designed to replace the less specific BSD License, and provides practical guidance for those who may redistribute software under the Apache License.

The redistribution requirements section of the Apache License is the section that most frequently uses the defined term, "Derivative Work". This is likely due to the fact that most of the distribution requirements (e.g. the requirement to identify modified files) only become relevant with respect to derivatives. What is interesting though, is that the "Derivative Work" definition seems to be based on US statutory law (the US Copyright Act), but with modifications.

The Apache License definition omits the examples in the US Copyright Act and combines the two US Copyright Act sentences into a conjunctive requirement: the work must be both a "work based on" the original work and the modifications as a whole must represent an original work of authorship to be a "Derivative Work". ${ }^{19}$ This definition still defers to the law to answer important questions like whether the modifications are significant enough to constitute a derivative work, so it is not entirely clear what advantage the editing of the statutory definition provides. The second sentence in the definition is more helpful in guiding interpretation, as it sets out technical rules excluding certain software combinations from the derivative work analysis.

As a non-copyleft licence, the definition of "Derivative Work" is not of major significance. The Apache License confirms its non-copyleft status in the last clause of the redistribution section, clarifying that modifications and "Derivative Works" as a whole may be licensed under different licensing terms.

\section{Contributions}

16 http://www.mozilla.org/MPL/MPL-1.1.html [hereinafter Mozilla Public License]

17 Id.

18 Apache License, supra note 1

19 Id. 
The Apache License has a notable clause dealing with contributions. The contributions clause, along with the contributions definition, provides clarity to open source projects that use the Apache License with respect to code submitted to the project without any separate agreement or licence notice. Such code is deemed to be licensed under the Apache License unless the author marks it with "not a contribution". This is a practical feature of the Apache License, supporting a collaborative development model by removing licensing ambiguity with respect to informally submitted works. The clause is also flexible, expressly contemplating separate contribution agreements and permitting them to override the Apache License.

\section{Compatibility}

\section{Apache and GPLv2}

According the Free Software Foundation, the Apache License 2.0 is not compatible with GPLv2 due to "certain patent termination and indemnification provisions." ${ }^{20}$ Both the Apache Foundation and the Free Software Foundation have updated their statements about compatibility over time, and a previous Free Software Foundation statement mentioned only "certain patent termination" provisions. ${ }^{21}$ A potential incompatibility in this respect results from the Apache License's patent termination provision, which could be thought to restrict the licensee's rights. The GPLv2 expressly prohibits "further restrictions" on the rights granted in the GPLv2. ${ }^{22}$ The Apache Foundation provided a detailed analysis of this argument, which left the compatibility question open. ${ }^{23}$ However, the Free Software Foundation's website still has a simple statement that it believes the Apache License 2.0 to be incompatible with GPLv2. ${ }^{24}$ The current Apache Foundation statement does not directly challenge this, but instead defers to the judgement of Free Software Foundation, stating that "The Apache Software Foundation believes that you should always try to obey the constraints expressed by the copyright holder when redistributing their work." ${ }^{25}$ The analysis of whether the licences are compatible seems to hinge on an interpretation of GPLv2 rather than the Apache License, and authors of these licences seem to suggest that moving to GPLv3 is a better way to answer the compatibility question.

\section{Apache and GPLv3}

The issue of Apache compatibility was directly addressed in the drafting of GPLv3. ${ }^{26}$ GPLv3 permits certain additional conditions to be added, one of which addresses the indemnity clause of the Apache License. ${ }^{27}$ The indemnity clause applies when the a distributor offers additional

20 http://www.gnu.org/licenses/license-list.html [hereinafter FSF Statement]

21 For older Apache Foundation statements about Apache License and GPL compatibility, see http://web.archive.org/web/20060426193453/http://www.apache.org/licenses/GPL-compatibility.html [hereinafter Historic Apache Statement]

For older Free Software Foundation statements about Apache License and GPL compatibility, see http://web.archive.org/web/*/http://www.gnu.org/licenses/license-list.html

22 http://www.gnu.org/licenses/gpl-2.0.html

23 Historic Apache Statement, supra note 24

24 FSF Statement, supra note 23

25 http://www.apache.org/licenses/GPL-compatibility.html [hereinafter Apache Statement]

26 Free Software Foundation, GPLv3 Final Discussion Draft Rationale, p. 9 (available at http://gplv3.fsf.org/rationale)

27 Id. 
liability obligations to downstream recipients (e.g. a warranty or indemnity). ${ }^{28}$ The Apache License permits these, but requires that the entity offering these indemnify upstream "Contributors" against claims asserted "by reason of" the additional obligations. ${ }^{29}$ Interpreting the "by reason of" phrase caused the Free Software Foundation originally to view the clause as problematic, but a subsequent interpretation as "nothing broader or vaguer than 'directly as a result of" led to the Free Software Foundation's agreement of GPLv3 compatibility (with the applicable additional condition mentioned above). ${ }^{30}$ This view seems appropriate, as additional warranty or indemnity liability can be offered outside the context of the Apache License. For example, a licensor could offer a warranty as part of a negotiated commercial support agreement. The relevant clause of the Apache License seems designed only to protect the copyright holders from any additional liability imposed as a result of a commercial arrangement between a downstream licensee and its customer.

The Free Software Foundation made other changes to drafts of GPLv3 which addressed potential compatibility issues related to the trademark clause and patent termination clause of the Apache License. $^{31}$ With regard to the trademark clause, the Apache License expressly reserves the trademark rights of the licensor, and GPLv3 expressly permits supplementing the GPLv3 with terms declining to grant certain trademark rights. ${ }^{32}$ These clauses demonstrate a conscious and successful effort to achieve compatibility between GPLv3 and the Apache License.

\section{Conclusion}

The Apache License can be thought of as a newer iteration of permissive non-copyleft licencses like the BSD License, MIT License, and historical permission notice. In that role, the Apache License provides additional legal clarity to the permissive licensing model, and also adds new terms such as a patent termination provision. The Apache License is written in the context of US law, matching phrasing to the US Copyright Act and US Patent Act, which is helpful to increasing clarity with respect to licensing in the US.

Some of the Apache License terms which go beyond simpler licences in the non-copyleft family have lead to debates over its compatibility with other licences, but there is a consensus that GPLv3 and the Apache License 2.0 are compatible.

\section{About the author}

Andrew Sinclair is Legal Counsel at Canonical, the commercial sponsor of Ubuntu.

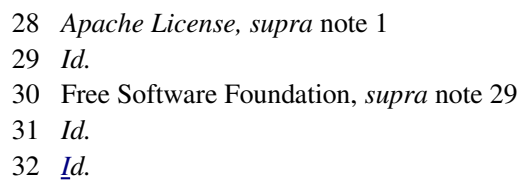




\section{Licence and Attribution}

This paper was published in the International Free and Open Source Software Law Review, Volume 2, Issue 2 (December 2010). It originally appeared online at http://www.ifosslr.org.

This article should be cited as follows:

Sinclair, Andrew (2010) 'Licence Profile: Apache License, Version 2.0', IFOSS L. Rev., 2(2), pp $107-114$

DOI: $\underline{10.5033 / \text { ifosslr.v2i } 2.42}$

Copyright 2010 Andrew Sinclair.

This article is licensed under a Creative Commons UK (England and Wales) 2.0 licence, no derivative works, attribution, CC-BY-ND.

As a special exception, the author expressly permits faithful translations of the entire document into any language, provided that the resulting translation (which may include an attribution to the translator) is shared alike. This paragraph is part of the paper, and must be included when copying or translating the paper.

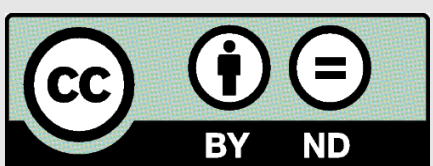


